\title{
P03-08. Protection against SIVmac239 challenge in a rhesus macaque model conferred by a doxycycline dependent attenuated SIVmac239
}

\author{
M Page*1, N Berry ${ }^{1}, M$ Robinson ${ }^{1}$, R Quartey-Papafio ${ }^{1}$, W Elsley ${ }^{1}, \mathrm{H}_{\text {Tudor }}{ }^{1}$, \\ A Das ${ }^{2}$, W Kleibeuker ${ }^{2}$, B Klaver ${ }^{2}$, B Li ${ }^{1}$, M Manoussaka $^{3}$, R Stebbings ${ }^{1}$, \\ $\mathrm{M}$ Cranage $^{3}$, B Berkhout ${ }^{2}$ and N Almond ${ }^{1}$
}

Address: ${ }^{1}$ National Institute for Biological Standards \& Control, Potters Bar, UK, ${ }^{2}$ Center for Infection and Immunity Amsterdam (CINIMA), Amsterdam, Netherlands and ${ }^{3}$ St. George's, University of London, London, UK

* Corresponding author

from AIDS Vaccine 2009

Paris, France. 19-22 October 2009

Published: 22 October 2009

Retrovirology 2009, 6(Suppl 3):P25 doi:10.1186/1742-4690-6-S3-P25

This abstract is available from: http://www.retrovirology.com/content/6/S3/P25

(C) 2009 Page et al; licensee BioMed Central Ltd.

\section{Background}

In the SIV/macaque model of HIV in man, live attenuated virus confers the most potent protection against wild-type virus challenge. We have developed a novel live attenuated (nef deleted) vaccine SIVmac239 (SIVrtTA) that is dependent on doxycycline for replication in vivo. Withdrawal of doxycycline prevents replication of SIVrtTA. We used this virus to vaccinate rhesus macaques to investigate the role of virus replication and persistence in protection against SIVmac239 challenge.

\section{Methods}

Two groups of 6 rhesus macaques were vaccinated with SIVrtTA and simultaneously dosed orally with doxycycline for 6 months. In one group (A) the dosing was stopped 8 weeks before challenge with SIVmac239 whilst in the other group dosing was maintained. A further control group (C) was vaccinated with attenuated SIVmac239 $\Delta$ nef with doxycycline dosing for 6 months and challenged with SIVmac239 along with a naive group (D) of 4 animals.

\section{Results}

Upon inoculation with the conditional replicating SIV, all animals became infected. Virus was recovered from blood and sequence analysis determined that there was no escape by the virus from antibiotic dependence. Virus RNA levels were determined from blood and followed a similar profile to other live attenuated SIV (with peak loads of 3-4 log titres at day 14 followed by a gradual decline). Two weeks following challenge with SIVmac239, plasma viral RNA levels were undetectable in one animal of group $\mathrm{A}$. The other animals in both groups (A and $\mathrm{B}$ ) vaccinated with SIVrtTA showed reduced RNA levels (range 2-6 log titres) compared to those ( $\sim 7$ log titre) of the naive control group (D). Three of the four group $C$ animals vaccinated with SIVmac239 $\Delta$ nef were protected.

\section{Conclusion}

Inoculation with SIVrtTA showed a marked vaccine effect. This new live attenuated virus will be a useful tool to identify the correlates of protection by this vaccine strategy. 